\title{
Title: CRISPR-mediated Multiplexed Live Cell Imaging of Nonrepetitive Genomic Loci
}

Authors: Patricia A. Clow ${ }^{1}$, Nathaniel Jillette ${ }^{1}$, Jacqueline J. Zhu ${ }^{1 *}$, Albert W. Cheng ${ }^{1,2,3,4 *}$

\section{Affiliations:}

${ }^{1}$ The Jackson Laboratory for Genomic Medicine, Farmington, CT 06032, USA

${ }^{2}$ The Jackson Laboratory Cancer Center, Bar Harbor, ME 04609, USA

${ }^{3}$ Department of Genetics and Genome Sciences, University of Connecticut Health Center, Farmington, CT 06030, USA

${ }^{4}$ Institute for Systems Genomics, University of Connecticut Health Center, Farmington, CT 06030, USA

$15 \quad *$ Correspondence to: jackie.zhu@jax.org or albert.cheng@jax.org

Abstract: Three-dimensional (3D) structures of the genome are dynamic, heterogeneous and functionally important. Live cell imaging has become the leading method for chromatin dynamics tracking. However, existing CRISPR- and TALE-based genomic labeling techniques have been hampered by laborious protocols and low signal-to-noise ratios (SNRs), and are thus mostly applicable to repetitive sequences. Here, we report a versatile CRISPR/Casilio-based imaging method, with an enhanced SNR, that allows for one nonrepetitive genomic locus to be labeled using a single sgRNA. We constructed Casilio dual-color probes to visualize the dynamic interactions of cohesin-bound elements in single live cells. By forming a binary sequence of multiple Casilio probes (PISCES) across a continuous stretch of DNA, we track the dynamic 3D folding of a $74 \mathrm{~kb}$ genomic region over time. This method offers unprecedented resolution and scalability for delineating the dynamic 4D nucleome.

One Sentence Summary: Casilio enables multiplexed live cell imaging of nonrepetitive DNA loci for illuminating the real-time dynamics of genome structures. 


\section{Main Text:}

Three-dimensional (3D) organization of the genome has a role in regulation of transcription, DNA replication, and DNA repair (1). Disruption of 3D genome structure and processes has been implicated in diseases $(2,3)$. Sequencing methods such as Hi-C and ChIA-PET $(4,5)$ can uncover genome-wide chromatin interactions in bulk cell populations but are unable to resolve intercellular heterogeneity or temporal dynamics. Fluorescence in situ hybridization (FISH) visualizes genomic loci in fixed specimens allowing for the observation of chromatin interaction in single cells but fails to track chromatin movement over time (6). A dynamic view of nuclear architecture and processes within the cell can be provided by live cell imaging techniques which have seen tremendous progress over recent years (7). Early studies depend on locus-specific integration of high-copy ( 256) LacO arrays to be bound by LacI-GFP fusion (8). However, these large insertions can disrupt genome folding and require laborious genome engineering for each locus of interest. Live cell imaging of native genomic loci is achievable with the use of programmable sequence-specific DNA binding proteins. Zinc finger proteins (ZFP) and transcription activator-like effectors (TALEs) linked to fluorescent proteins allow imaging of genomic loci in live cells, but the complicated cloning protocols limit their scalability $(9,10)$. The CRISPR/Cas systems offer versatile DNA binding proteins such as Cas9 that can be programmable by a guide RNA (gRNA) for a specific target and has revolutionized the field of genome engineering and genomics (11). Fluorescent proteins recruited by dCas9 or assembled on the gRNA scaffold offer easy-to-use platforms for live cell genomic imaging (12-17). However, current approaches have poor signal-to-noise ratios (SNR). Despite providing robust labeling of repetitive sequences using a single gRNA, imaging of nonrepetitive sequences requires simultaneous delivery of up to tens of gRNA to achieve sufficient signal, precluding 
broad adoption. We present here a CRISPR/Cas-based genomic imaging method with drastically enhanced SNR for labeling of a given nonrepetitive locus with one gRNA, enabling the monitoring of chromatin interaction and tracking of genome folding by multiplexed deployment of probes across a stretch of DNA.

We previously engineered a modular multitasking CRISPR/Cas-based platform called Casilio by combining $\underline{\text { Cas } 9}$ and engineered Pumilio/FBF (PUF)-tethered effectors (18). Effectors are tethered by the PUF RNA binding domain to assemble on an extended gRNA inserted with one of more copies of specific 8-mer motifs. PUF domains contain peptide subunits that can each be programmable to recognize an RNA-base by changing amino acids contacting the RNA, thereby allowing designed PUF domains to bind different 8-mer sequences $(19,20)$. Casilio capitalizes on the versatility of PUF domains to allow simultaneous and orthogonal delivery of effector functions to distinct genome loci, as well as multimerization of effector molecules, for efficient epigenetic editing $(18,21)$. In the case of genomic labeling, we demonstrated not only greatly enhanced SNR but also simultaneous dual color labeling of telomeric and centromeric 15 repeats $(18)$.

In this study, we further developed Casilio for imaging nonrepetitive sequences. We first labeled nonrepetitive sequences within the MUC4 gene on chromosome 3 which was previously labeled by dCas9-EGFP direct fusion by tiling $>30$ gRNAs over the region (12). We chose ten gRNAs located within a $5 \mathrm{~kb} M U C 4$ region and tested them with Casilio in pairwise 20 combinations by labeling one locus with sgRNA-15xPBSc/Clover-PUFc and another with sgRNA-15xPBSa/mRuby-PUFa in U2OS cells (Fig. 1A). Colocalization of these nonrepetitive loci confirmed that Casilio can use a single gRNA to visualize a nonrepetitive sequence with high SNR and specificity (Fig. 1B to H). 
Since dual-color labeling by Casilio produced largely overlapping fluorescent foci for pairs of genomic loci within $5 \mathrm{~kb}$ distance, we next sought to identify the resolution limit of the technique. To this end, we designed gRNAs to target nonrepetitive loci at increasing distances from MUC4 locus 0 (Fig. 2A). As expected, physical distances as well as cellular heterogeneity between pairs increase with larger genomic distances (Fig. 2B and C). Loci separation became apparent at approximately $28 \mathrm{~kb}$ distance, demonstrating that the Casilio imaging approach offers a resolution down to $28 \mathrm{~kb}$ using our spinning disk confocal microscope.

To test whether Casilio can be applied to study the dynamics of chromatin interactions in live cells, we selected two chromatin interactions from published cohesin (RAD21) ChIA-PET dataset (ENCSR110JOO, Michael Snyder lab) with 53kb and 362kb genomic separations. We then designed a pair of one-copy gRNAs to bind next to RAD21 peaks for each interaction using a combination of ChIA-PET2, JACKIE and Cas-OFFinder (22-24) (Fig. 3A). Live cell microscopy of ARPE-19 cells transfected with dual-color Casilio probe pairs revealed highly dynamic chromatin interactions at second timescales (Fig. 3B-G). This demonstrates that Casilio can image pairwise interactions of nonrepetitive sequence elements with higher spatial and temporal resolution than preiously possible.

Imaging specific interactions of non-coding elements such as enhancers and promoters provides new insights into gene regulation, while visualizing a continuous stretch of genomic region will improve our understanding of structural folding dynamics and illuminate the process 20 of chromatin loop formation. Given that Casilio can image each nonrepetitive locus with a single gRNA, we next explored the possibility of simultaneously imaging multiple nonrepetitive loci, with the specific goal of tracking the "live" structure of a continuous genomic region. We call this technique, where sequential Casilio probes are deployed across a stretch of genomic DNA, 


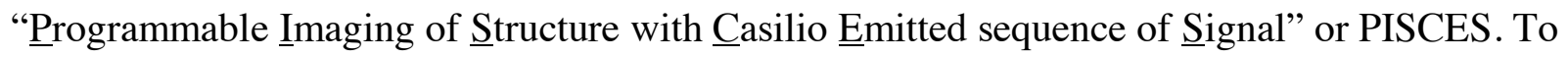
reduce the number of plasmids for transfection, we first constructed a plasmid with an array of five gRNAs targeting MUC4 loci $0,28 \mathrm{~kb}, 44 \mathrm{~kb}, 58.5 \mathrm{~kb}$, and $74 \mathrm{~kb}$ with alternating $15 \mathrm{xBSc}$ or 15xPBS9R scaffolds (Fig. 4A). Five clustered fluorescent foci (three green plus two magenta) were observed in HEK293T cells (Fig. 4B and C) and ARPE-19 cells (Fig. 4D and E) transfected with the pentameric gRNA arrays. These results demonstrated the use of a dual-color combination to encode a sequence of PISCES probes for illuminating the dynamic folding of a $\sim 70 \mathrm{~kb}$ genomic region in live cells.

In this study, we present a CRISPR/Cas-based method for live cell fluorescence imaging of nonrepetitive genomic loci with low gRNA requirement (one gRNA/locus) and high spatiotemporal resolutions ( $<28 \mathrm{~kb}$ at second timescales). We applied Casilio to visualize the dynamic interactions of two pairs of cohesin-bound elements in native, unmodified chromosomes. Using a binary code of two fluorescent proteins (PISCES), we showed that folding of a continuous stretch of DNA can be imaged over time. Casilio imaging probes and 15 PISCES revealed the highly heterogeneous and dynamic nature of chromatin interaction and folding, underlining the need for studying the 4D nucleome with high spatiotemporal resolutions. The reduction of gRNA requirement of Casilio-based imaging compared with previously published CRISPR-based approaches not only significantly reduces the technical challenge of using live cell imaging to study chromatin interactions in hard-to-transfect cells, but it also 20 simplifies future design of genome-wide gRNA libraries for imaging. Future development of the Casilio imaging toolkit should include higher-density PISCES probes with an expanded palette of fluorescent proteins. The multiplexing and expandable nature of Casilio in adapting new effector functions (18), in combination with the advance in genomic imaging demonstrated here, 
will enable the "on-the-fly" and "plug-and-play" perturbation of the (epi)genome (e.g., using our activator and repressor modules) and the concomitant read-out of dynamic 3D chromatin interactions, thereby provinding unprecedented flexibility and power for the study of the 4D nucleome.

Acknowledgments: We thank the Jackson Laboratory Microscopy Service for microscope usage, Jane Cha (JAX Creative) for graphic design, and Jackson Laboratory Research Program Development for help with manuscript editing. Funding: This work is supported by internal funds provided by the Jackson Laboratory as well as grants from the National Cancer Institute (P30CA034196) and the National Human Genome Research Institute (R01-HG009900). Author contributions: J.J.Z. and A.W.C. conceived the study. P.A.C., J.J.Z, and A.W.C. designed the experiments. P.A.C., N.J., J.J.Z., and A.W.C. performed the experiments. P.A.C. analyzed the data. P.A.C., J.J.Z., and A.W.C. wrote the manuscript with help from all authors. Competing interests: A patent application has been filed on the invention. 


\section{Figure Legends}

Fig. 1. Casilio live-cell imaging of pairs of nonrepetitive genomic loci within $5 \mathbf{k b}$. (A)

Schematic of Casilio probes for visualizing pairs of genomic loci with a gRNA targeting one locus (horizontal bar) with $15 \times \mathrm{PBSc}$ recruiting Clover-PUFc and a gRNA targeting another locus within $5 \mathrm{~kb}$ (vertical bar) with $15 \mathrm{xPBSa}$ recruiting mRuby2-PUFa. Letters in grid are positioned according to the two gRNAs used and indicate the corresponding microscopic images. (B to $\mathbf{H})$ Representative images of U2OS/dCas9 cells transfected with Clover-PUFc, mRuby-PUFa and the gRNA pairs as indicated in the grid. Scale bars, $5 \mu \mathrm{m}$.

\section{Fig. 2. Casilio live-cell imaging of pairs of nonrepetitive genomic loci with increasing}

distance. (A) Schematic of Casilio probes for visualizing MUC4 locus 0, labeled with $15 \times \mathrm{PBSa} / \mathrm{mRuby}-\mathrm{PUFa}$, and another locus, labeled with $15 \times \mathrm{PBSc} / \mathrm{Clover}-\mathrm{PUF}$, at increasing distances from locus 0. (B) A plot of 3D distances (in $\mu \mathrm{m}$ ) between observed fluorescent foci pairs versus genomic distances (in $\mathrm{kb}$ ) of the probe pairs in U2OS cells. Average distances marked by orange bars. (C) Representative images of U2OS cells labeled with Casilio probe 15 pairs with the indicated genomic distances. Scale bars, $5 \mathrm{~mm}$.

Fig. 3. Casilio live-cell imaging of chromatin interactions. (A) Schematic of Casilio probes for visualizing chromatin interactions mediated by cohesin (RAD21) with a gRNA targeting locus A (genomic 5' anchor) with 15xPBSc recruiting Clover-PUFc and a gRNA targeting locus B (genomic 3' anchor) with 15xPBS9R recruiting PUF9R-mRuby2. (B) WashU EpiGenome

20 Browser view of chr3:187318256-187680546 loop with anchors $367 \mathrm{~kb}$ apart on chromosome 3. Locus A and locus B indicate the gRNA binding locations. (C) Representative time-lapse images of chr3:187318256-187680546 loop anchors, Locus A near MASPl (green) and Locus B near BCL6 (magenta), in ARPE-19 cells. Image on the left shows the whole nucleus at time 0 (Scale 
bar, $5 \mu \mathrm{m}$ ). Image strips on the right show Pair 1 (upper) and Pair 2 (lower) at the indicated time point (Scale bars, $1 \mu \mathrm{m}$ ). (D) Pairwise 3D distance (in $\mu \mathrm{m}$ ) of fluorescent foci for Pair 1 and Pair 2 over time. (E) WashU EpiGenome Browser view of chr17:40302616-40355921 loop with anchors $55 \mathrm{~kb}$ apart on chromosome 17. (F) Representative time-lapse images of chr17:40302616-40355921 loop anchors, Locus A in CDC6 (green) and Locus B in RARA (magenta), in ARPE-19 cells. Image on the left shows the whole nucleus at time 0 (Scale bar, 5 $\mu \mathrm{m})$. Image strips on the right show Pair 1 (upper) and Pair 2 (lower) at the indicated time point (Scale bars, $1 \mu \mathrm{m}$ ). (G) Pairwise 3D distance (in $\mu \mathrm{m}$ ) of fluorescent foci for Pair 1 and Pair 2 over time.

Fig. 4. PISCES - Casilio live-cell imaging of five sequential nonrepetitive loci. (A) Schematic of Casilio/PISCES sequential 0-28kb-44kb-58.5kb-74kb probe for visualizing a $74 \mathrm{~kb}$ genomic region chr3:195,735,394-195,809,539 comprising of three gRNAs targeting three locations $(0$, $44 \mathrm{~kb}, 74 \mathrm{~kb}$ ) with $15 \times \mathrm{PBSc}$ recruiting Clover-PUFc and two gRNAs targeting two locations (28 kb, 58.5 kb) with 15xPBS9R recruiting PUF9R-mRuby2. (B and D) Representative time-lapse images of chr3:195,735,394-195,809,539 imaged with 0-28kb-44kb-58.5kb-74kb

Casilio/PISCES probes in HEK293T(B) and ARPE-19(D) cells. (Scale bars, 5 $\mu \mathrm{m}$ ). (C and E) 3D models of marked fluorescent clusters (see B and D) at time 0 in HEK293T(C) and ARPE-19(E) cells and the microscopic maximum intensity projections from the $\mathrm{x}-\mathrm{y}, \mathrm{y}-\mathrm{z}$, and $\mathrm{x}-\mathrm{z}$ perspectives. 

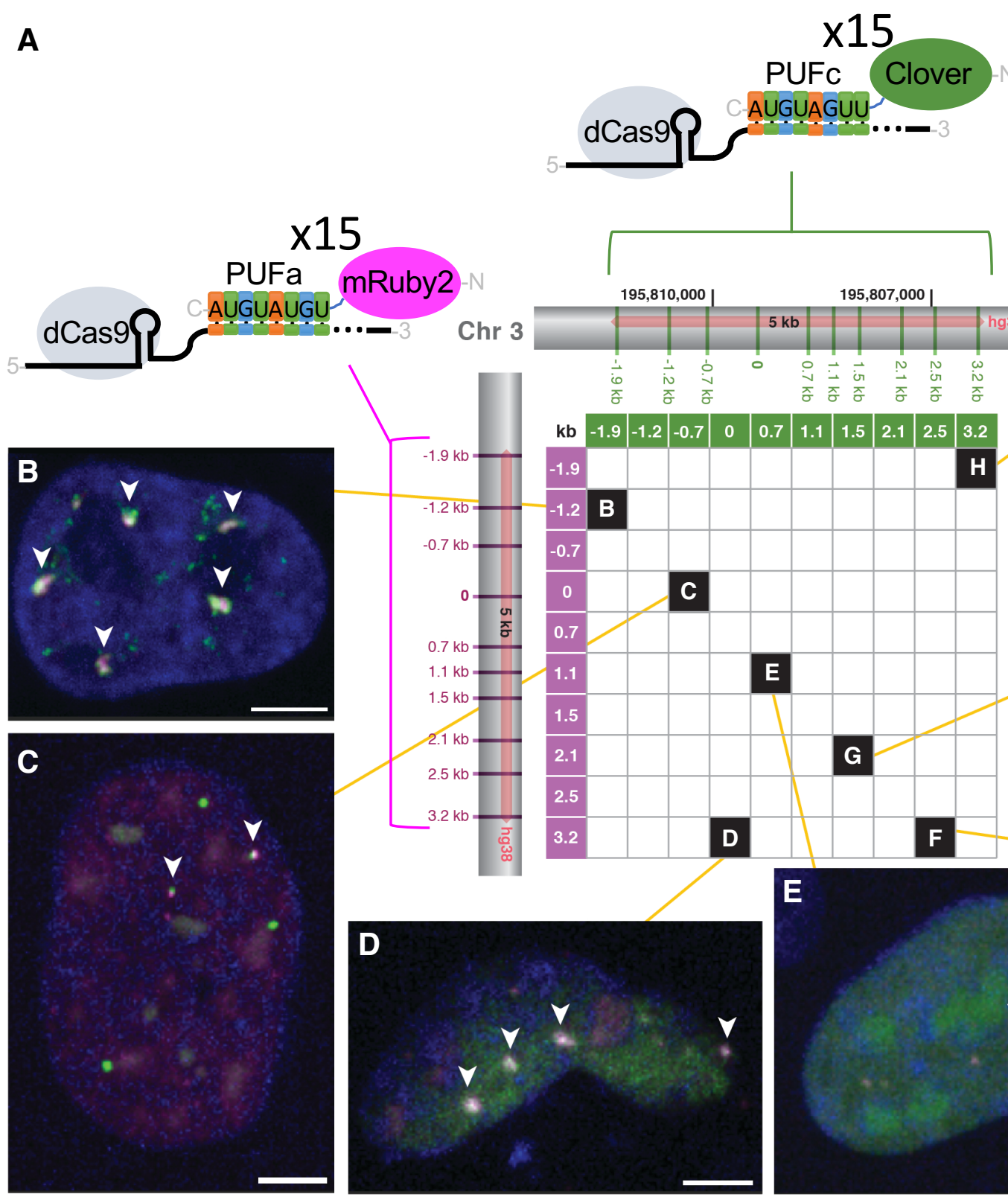

x15 
bioRxiv preprint doi: https://doi.org/10.1101/2020.03.03.974923; this version posted March 5, 2020. The copyright holder for this preprint

A (which was not certified by pegreview) is the author/funder. All rights reserved. No reuse alpqug without permission.

B

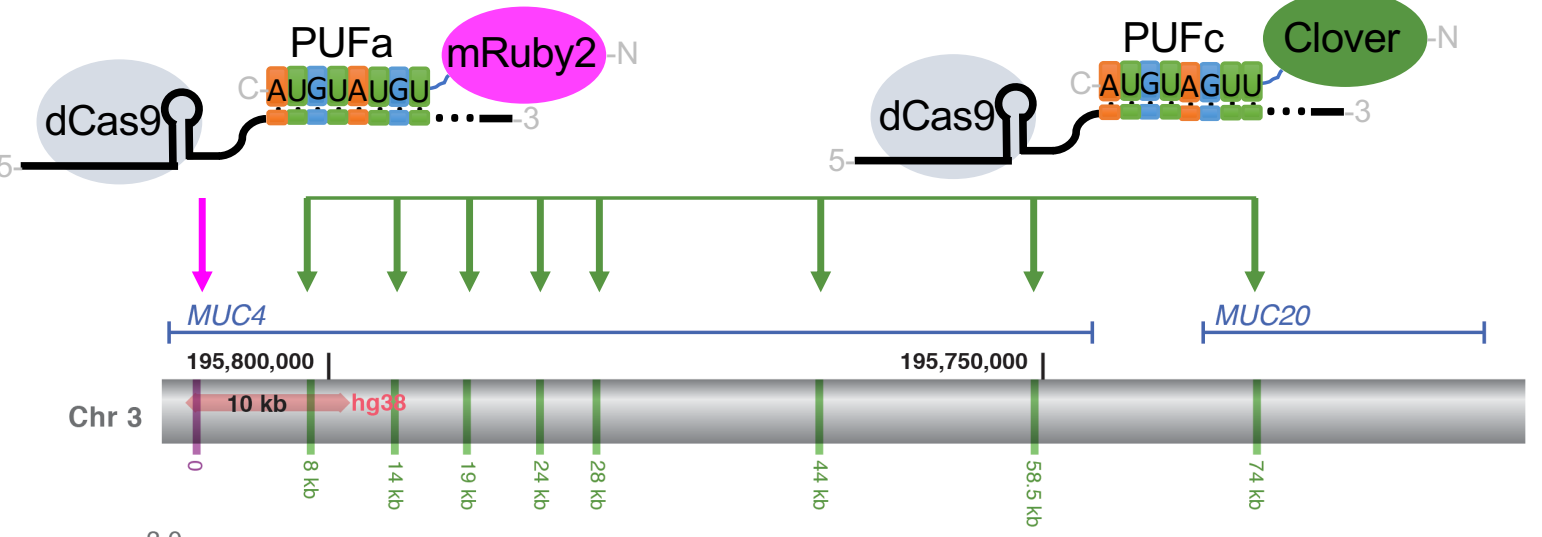

B

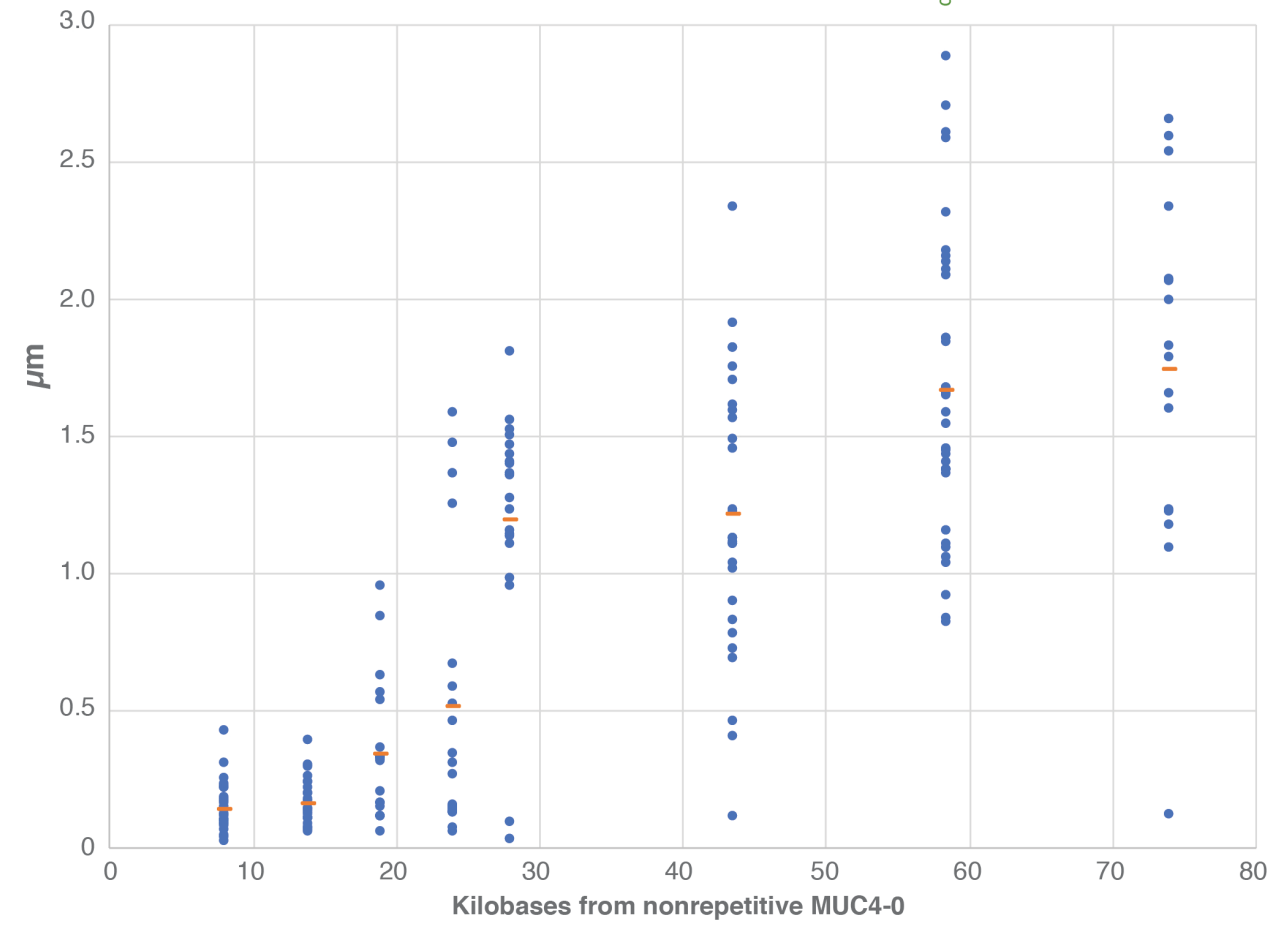

C
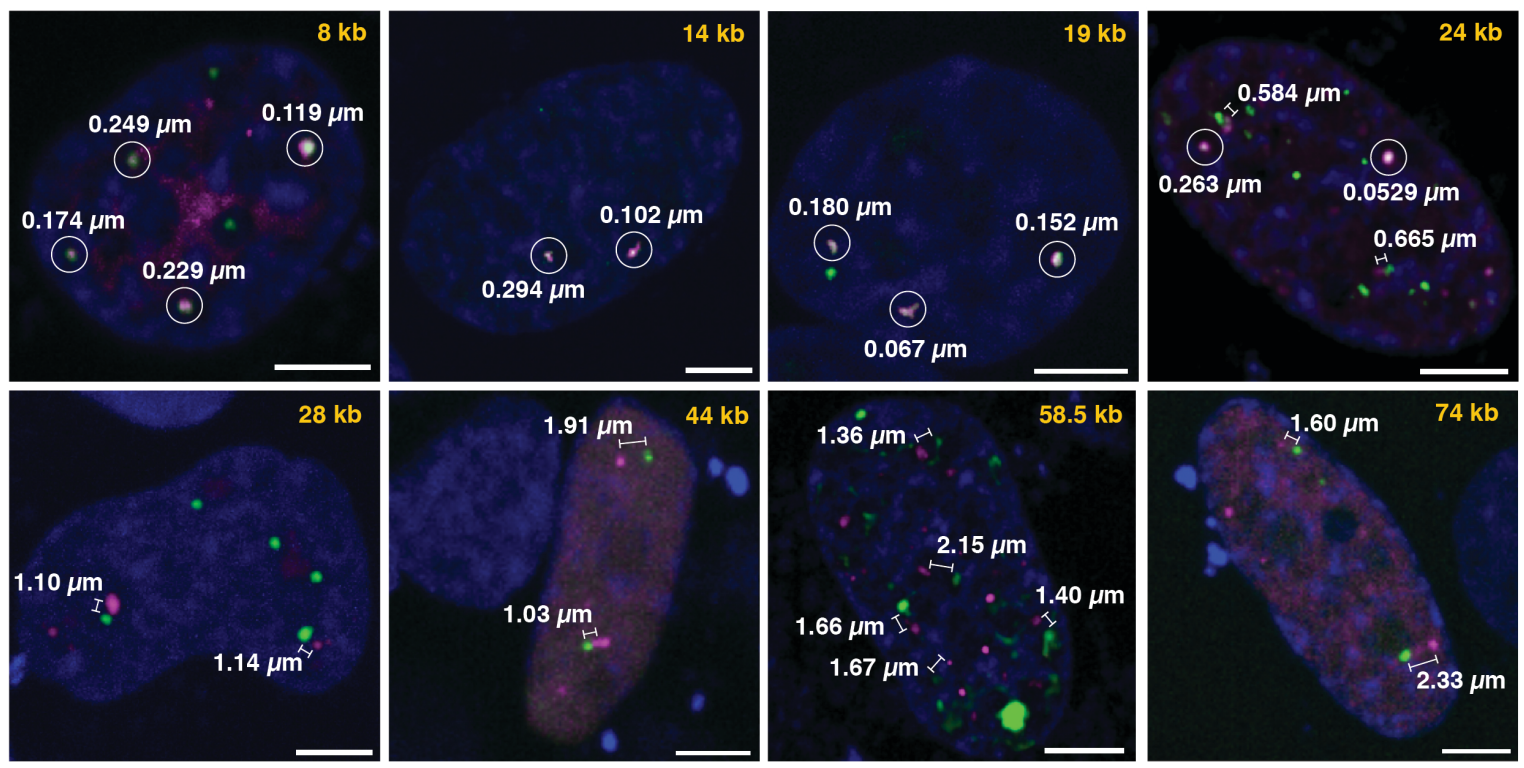

(which was not certified by peer review) is the author/funder. All rights reserved. No reuse allowed without permission.
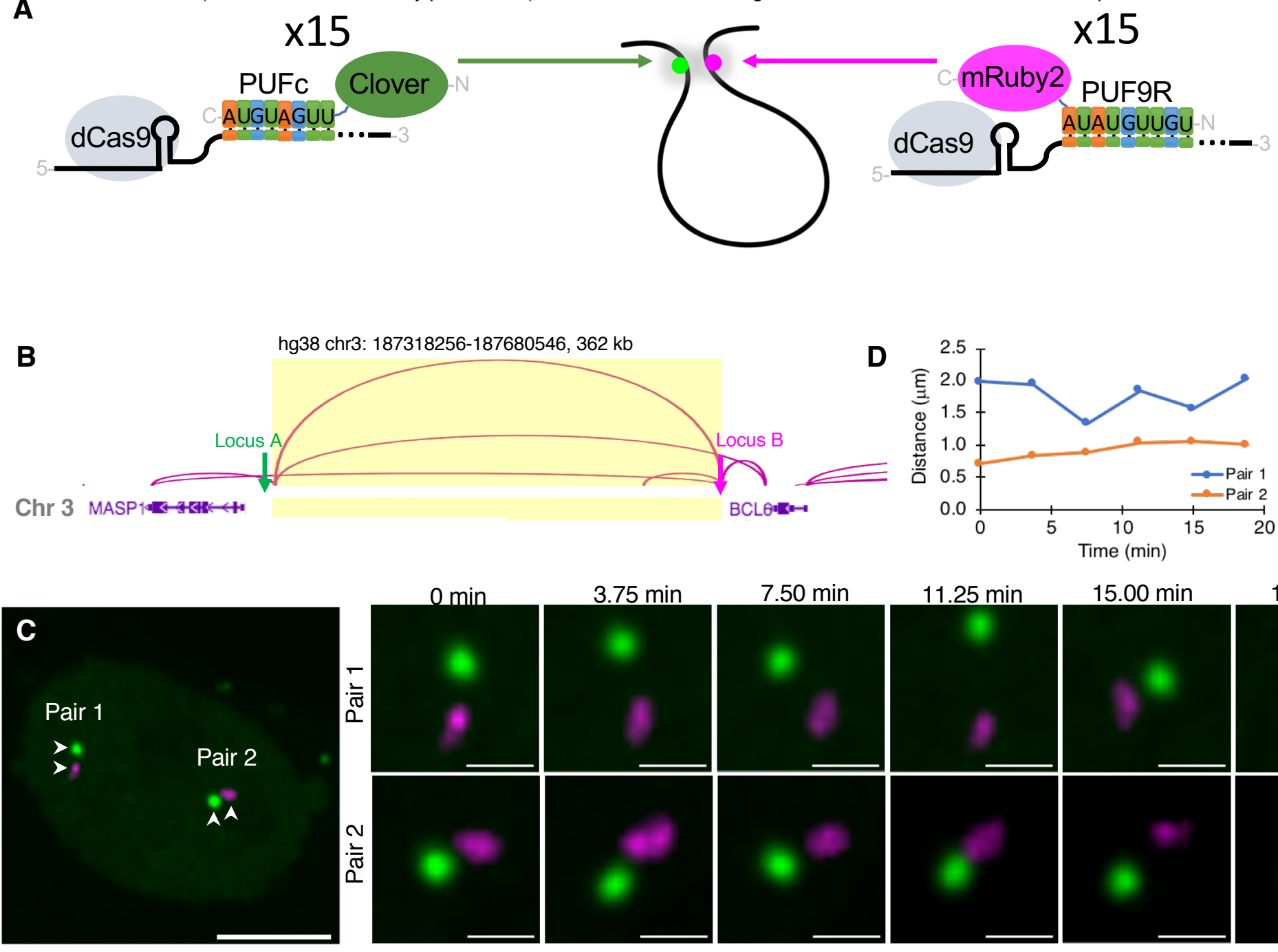

$18.75 \mathrm{~min}$

$\mathbf{E}$
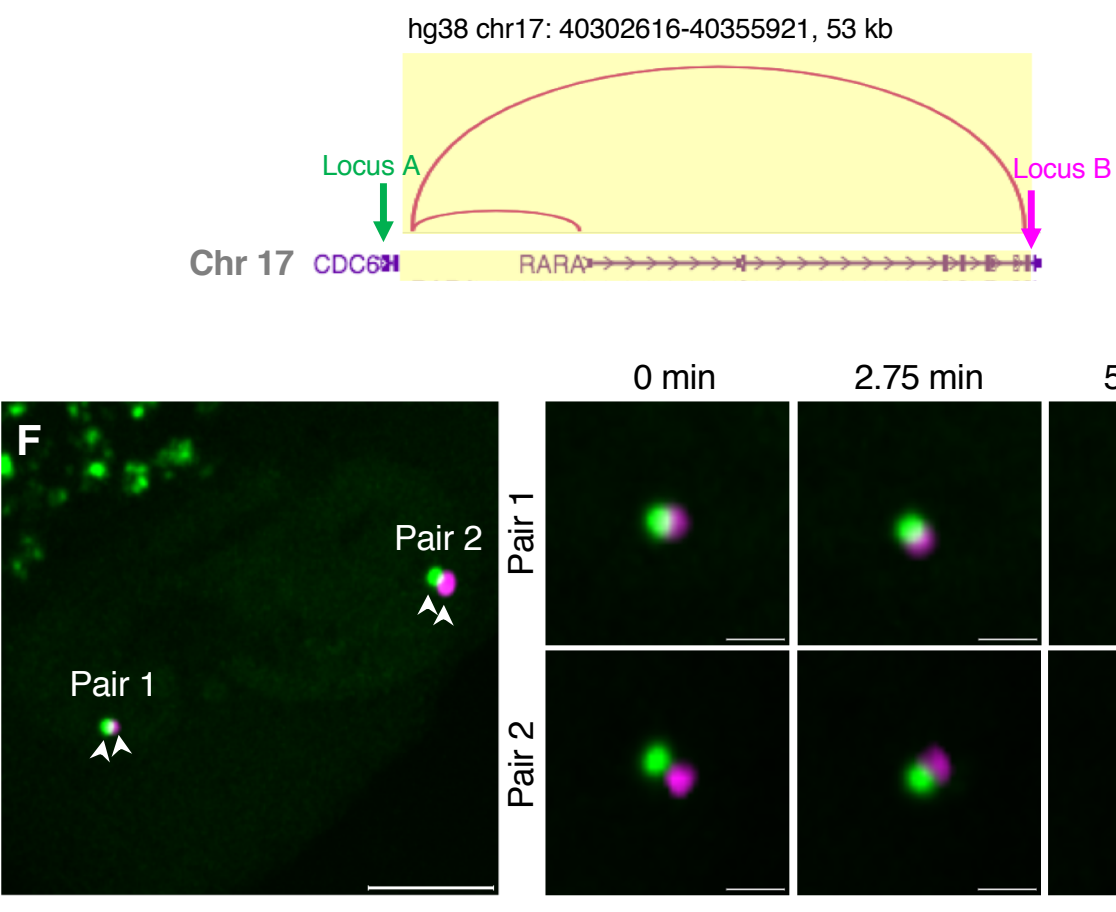

\section{$2.75 \mathrm{~min}$}

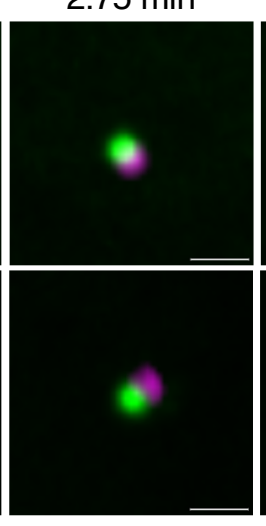

G
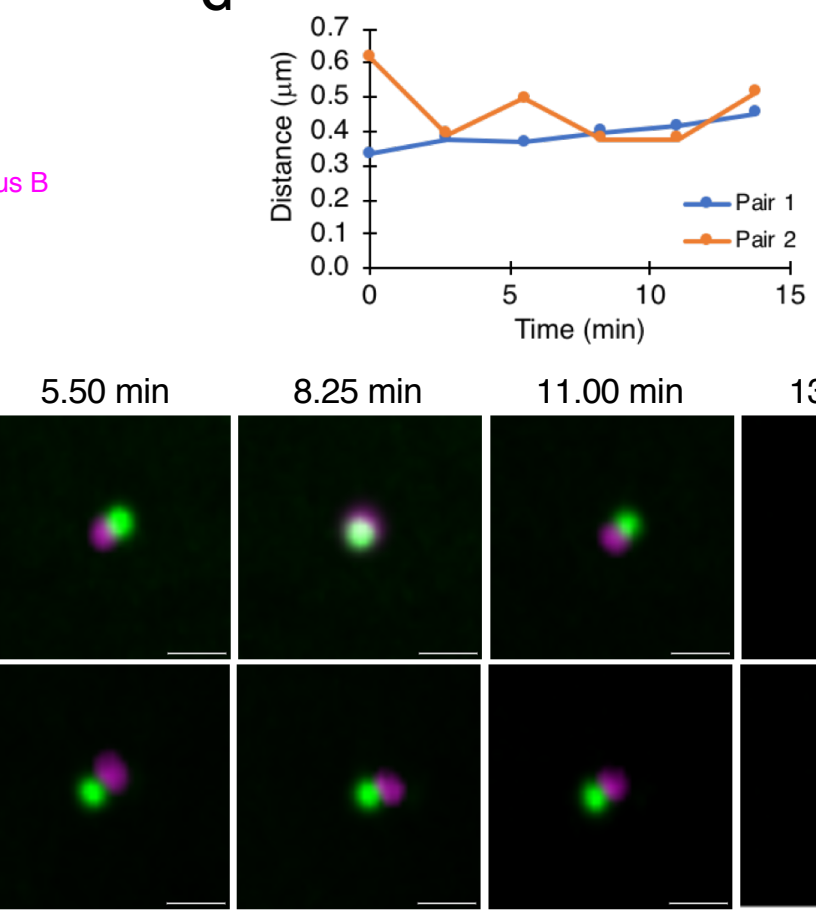

\begin{abstract}
$8.25 \mathrm{~min}$
\end{abstract}

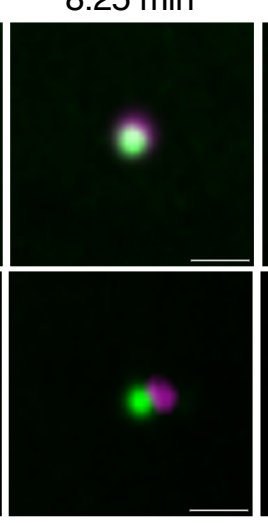

$11.00 \mathrm{~min}$

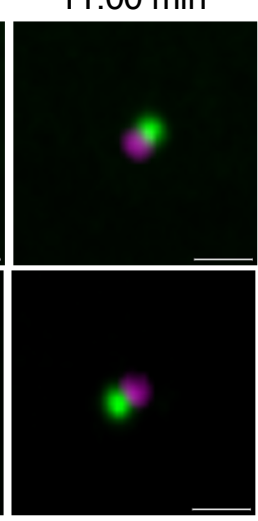

$13.75 \min$ 
bioRxiv preprint doi: https://doi.org/10.1101/2020.03.03.974923; this version posted March 5, 2020. The copyright holder for this preprint

A

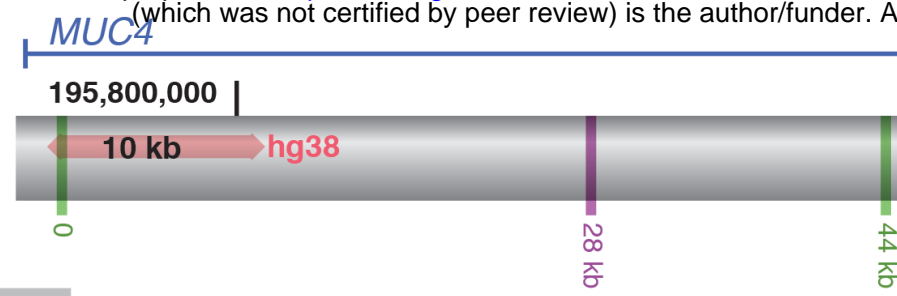

\section{B HEK293T}

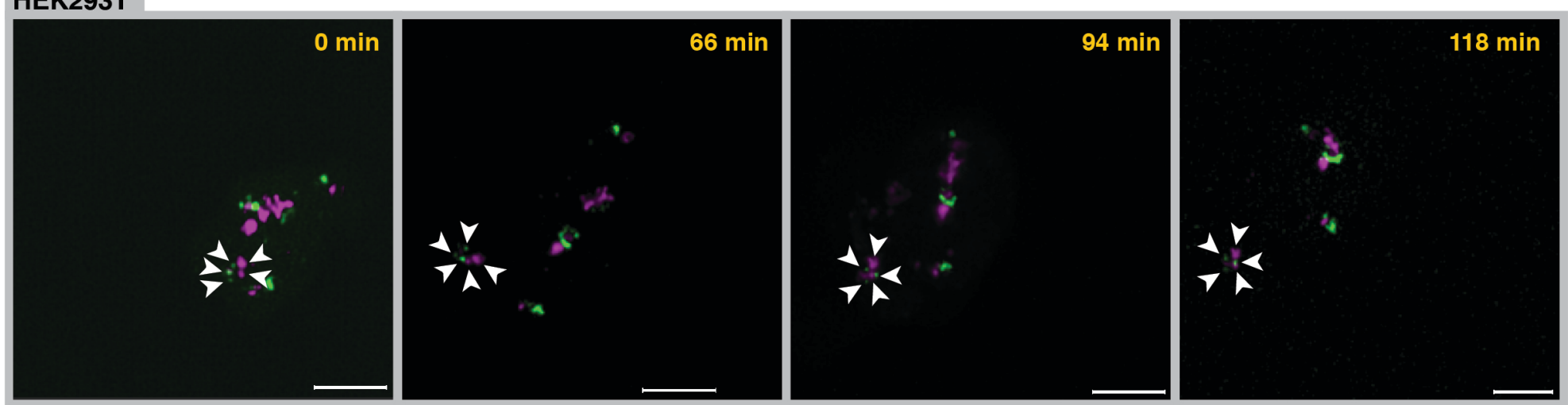

C

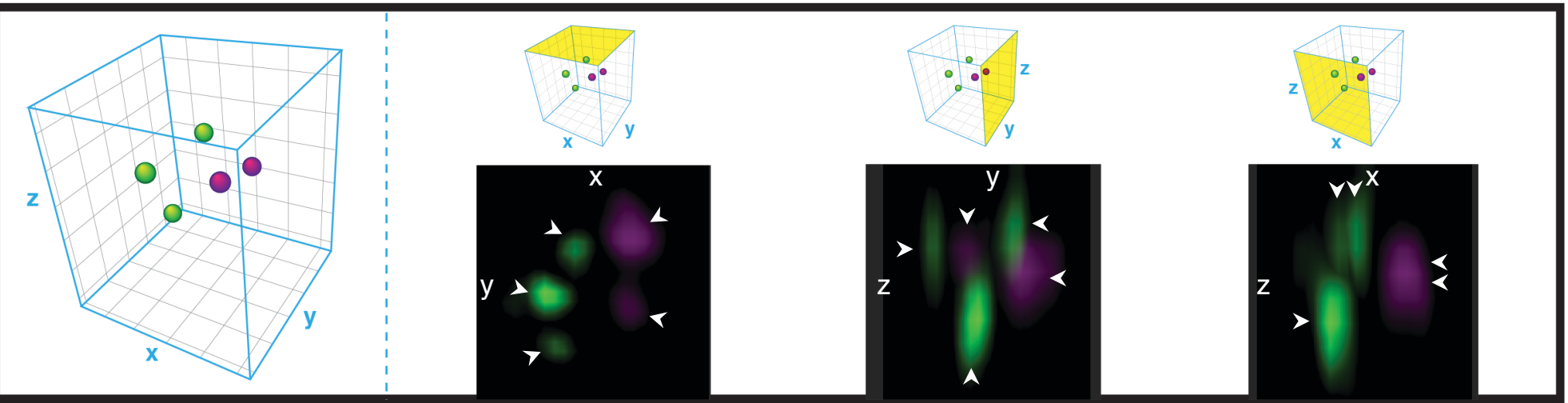

D ARPE-19

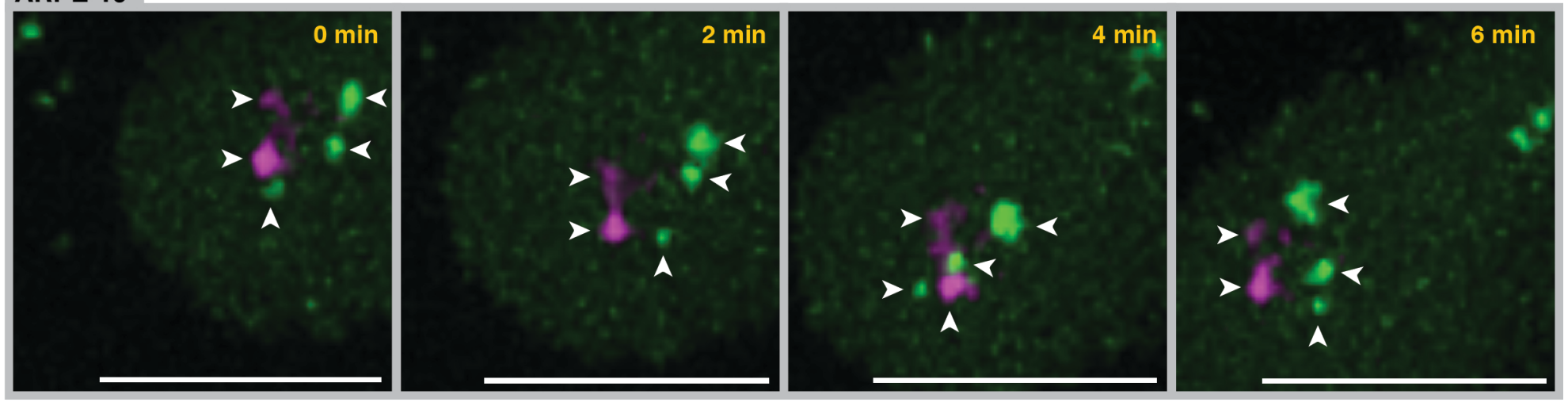

E

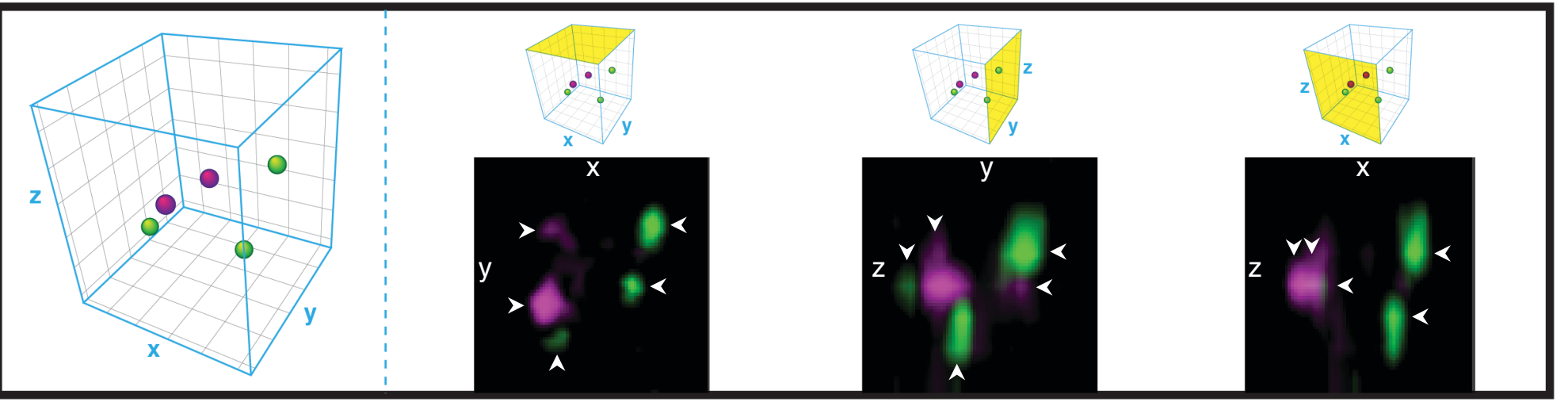

Figure 4 


\section{Materials and Methods}

\section{Design of chromatin interaction imaging experiments (Figure 3)}

Fastq files of ARPE-19 RAD21 ChIA-PET experiment (ENCSR110JOO) was downloaded from

ENCODE database, and processed by ChIA-PET2 (22). Interactions were ranked by PET count. Top-ranked interactions with different PET distances were selected. To avoid interference of CTCF binding, design regions were selected by shifting external to PET regions. JACKIE was then used to identify unique 1-copy CRISPR sites in the hg38 genome overlapping design regions of selected loops (23) and further filtered for specificity by Cas-OFFinder (24). ChIAPET loops are displayed on WashU EpiGenome Browser (https://epigenomegateway.wustl.edu/).

\section{Cloning}

The lentiviral dCas9 expression plasmid (lenti-dCas9-Blast) was generated by PCR-based mutagenesis of lentiCas9-Blast plasmid (Addgene \#52962). Clover and mRuby2 fused with PUF RNA-binding domain were previously described: pAC1447 (Clover_PUFc) (Addgene \#73689) and pAC1448 (mRuby2_PUFa) (Addgene \#73690). A new PUF9R-tethered mRuby2 was created by a combination of PCR (from IDT gBlocks) and ligation cloning placing mRuby2 downstream of PUF9R. These PUF-fluorescent protein fusions contain nuclear localization signal (NLS) for their localization in the nucleus. or pAC1430-pX-sgRNA-15xPBSc (Addgene \#71930), pCR8-sgRNA-15xPBSa, pCR8-sgRNA15xPBSc, pCR8-sgRNA-15xPBS9R via an oligo-annealing protocol (25). For generating a 5-in- 
1 gRNA array plasmid, multiple cloning steps were used to insert gRNA-PBS one by one using a combination of PCR and SLIC (26).

All plasmids were subjected to restriction diagnostic tests and sequenced to ensure correct cloning. Plasmids will be available on Addgene.

Target sequences for sgRNA

Designs from a previous study (12):

MUC4 -1.9 kb: GAAGAGTGGAGGCCGTGCGCGG

MUC4 -1.2 kb: GCAAGCAAGGGAAGCGACAAGG

MUC4 -0.7 kb: GATGTTTCAGGACTAGGCTGA

10 MUC4 locus 0: GAGCTGGGCCAGGAGAGGAGA

MUC4 0.7 kb: GAGGGGTCTGTGGAGAGTTT

MUC4 1.1 kb: GTGGAGACAGGGTTGGCCAAGC

MUC4 1.5 kb: GGCTTGGTGTATTCAGAATG

MUC4 $2.1 \mathrm{~kb}:$ GACAGAGTTTCTCTCTGTCCCCC

15

MUC4 2.5 kb: GTAGAGATGCCGCCCCGCCC

MUC4 3.2 kb: GACAAGTCAGGAAGGGCCCTGTG

New designs from this study used in Figures 2 and 4:

MUC4 8 kb: GCTCCCCAAGTTTATGCGTT

MUC4 14 kb: GGCGGTTCCAGCTTTTTGCA

MUC4 19 kb: GTTGATGTTGTAACCGGTGTGA

MUC4 24 kb: GCATCGGTGTCATGAAGAGCGG

MUC4 28 kb: GCCGGTGACAGGAAGAGTGC 
MUC4 44 kb: GAATCCACCAAGAAGAGGCCC

MUC4 58.5 kb: GAAGTCAATGACCGGGCCCCGA

MUC4 74 kb: GGATTGGAGCAAAGAAGTGGTG

MUC4 94 kb: GACAGACTCATCCCCAGGAC

New designs used in Figure 3 for RAD21 loops in ARPE-19 cells:

Figure 3B-D: Locus A near MASP1: GGGTAAGAAGCCACTAGGGT

Figure 3B-D: Locus B near BCL6: GCATAGCCGCATTTGAAAGC

Figure 3E-G: Locus A in CDC6: GTGAATAAGACCAACCCTCT

Figure 3E-G: Locus B in RARA: GCGGGTGATCACGCTGAAGA

10

\section{$\underline{\text { Cell culture }}$}

Human osteosarcoma U2OS cells $\left(\mathrm{ATCC}^{\circledR} \mathrm{HTB}^{\mathrm{N}}-6^{\mathrm{TM}}\right.$ ) and human embryonic kidney HEK293T cells (ATCC ${ }^{\circledR}$ CRL-3216 ${ }^{\mathrm{TM}}$ ) were cultivated in Dulbecco's modified Eagle's medium (DMEM) (Sigma) with 10\% fetal bovine serum (Lonza), 4\% Glutamax (Gibco), 1\% Sodium

15 Pyruvate (Gibco) and penicillin-streptomycin (Gibco). Human retinal pigment epithelial ARPE19 cells $\left(\mathrm{ATCC}^{\circledR}\right.$ CRL-2302 ${ }^{\mathrm{TM}}$ ) were cultivated in DMEM/F12 (Gibco) with 10\% fetal bovine serum (Lonza), and 1\% penicillin-streptomycin (Gibco). Incubator conditions were humidified $37^{\circ} \mathrm{C}$ and $5 \% \mathrm{CO}_{2}$. Cell lines expressing constitutive dCas 9 were generated by transducing cells lentiviruses prepared from a lenti-dCas9-Blast plasmid, followed by Blasticidin selection.

\section{$\underline{\text { Transfection }}$}

U2OS/dCas9 cells were seeded at density of 55,000-125,000 cells/compartment in a 35 mm 4-compartment CELLview cell culture dish (Greiner Bio-One) 24 hours before transfection. Cells were transfected with 75-125 ng of sgRNA plasmid DNA containing 15 Pumilio Binding 
Sites (15xPBS), 10-25 ng of Clover-PUF fusion plasmid DNA, and 15-25 ng of mRuby2-PUF fusion plasmid using $0.5 \mu \mathrm{l}$ Attractene (Qiagen) or 0.75-1 $\mu$ l Lipofectamine 3000 (Invitrogen). Media was changed at 24 hours post-transfection.

HEK293T/dCas9 cells were seeded at density of 200,000 cells/compartment in $35 \mathrm{~mm} 4$ compartment CELLview cell culture dish (Greiner Bio-One) 18 hours before transfection. Cells were transfected with $50 \mathrm{ng}$ of sgRNA-15xPBS plasmid DNA, $10 \mathrm{ng}$ of Clover-PUF fusion plasmid DNA, and $75 \mathrm{ng}$ of PUF-mRuby2 fusion plasmid DNA using $0.75 \mu$ Lipofectamine 3000 (Invitrogen). Media was changed at 24 hours post-transfection.

ARPE-19/dCas9 cells were seeded at density of 50,000-110,000 cells/compartment in 35 mm 4-compartment CELLview cell culture dish (Greiner Bio-One) 6-28 hours before transfection. Cells were transfected with 200-600 ng of sgRNA-15xPBS plasmid, and 5-40 ng of Clover-PUF fusion plasmid DNA, and 30-700 ng of PUF-mRuby2 fusion plasmid DNA using 1.5-1.7 $\mu$ l Lipofectamine LTX (Invitrogen). Media was changed at 24 hours post-transfection.

Imaging was performed at 25-71 hours post-transfection. Prior to imaging, cells were stained with $0.5-1.0 \mu \mathrm{g} / \mathrm{ml}$ Hoechst 33342 prepared in cell culture media for 30-60 minutes, followed by two media washes. Images were acquired with the Dragonfly High Speed Confocal Platform 505 (Andor) using a Zyla sCMOS camera or an iXon EMCCD camera and a Leica HC PL APO 63x/1.47NA OIL CORR TIRF objective mounted on a Leica DMi8 inverted microscope equipped with a live-cell environmental chamber (Okolab) at humidified $37^{\circ} \mathrm{C}$ and 5\% $\mathrm{CO}_{2}$. Imaging mode was Confocal $40 \mu \mathrm{m}$ or Confocal $25 \mu \mathrm{m}$. Hoechst images were acquired with a $200 \mathrm{~mW}$ solid state $405 \mathrm{~nm}$ laser and 450/50 nm BP emission filter. Clover images were 
acquired with a $150 \mathrm{~mW}$ solid state $488 \mathrm{~nm}$ laser and 525/50 nm BP emission filter. mRuby2 images were acquired with a $150 \mathrm{~mW}$ solid state $561 \mathrm{~nm}$ laser and 620/60 nm BP emission filter. Z-series covering the full nucleus was acquired at 0.13-1.0 $\mu \mathrm{m}$ step size. For time-lapse imaging, the Z-series was acquired at 0.2-0.4 $\mu \mathrm{m}$ step size. Images are a maximum intensity projection of Z-series.

$\underline{\text { Image analysis }}$

Imaris (Bitplane) image analysis software was used to measure spot distances. Z-series acquired at 0.19-0.5 $\mu \mathrm{m}$ step size was used. For each channel, spots were segmented based on maximum intensity in the 3D volume of the nucleus. Measurement points were set to intersect with the center of the spot object. With line mode set as pairs, distances between loci pairs in the 3D volume were measured from a spot in one channel to the closest spot in the other channel. 


\section{References and Notes:}

1. P. G. Maass, A. R. Barutcu, J. L. Rinn, Interchromosomal interactions: A genomic love story of kissing chromosomes. The Journal of cell biology 218, 27-38 (2019).

2. A. Mishra, R. D. Hawkins, Three-dimensional genome architecture and emerging technologies: looping in disease. Genome medicine 9, 87 (2017).

3. A. Krumm, Z. Duan, Understanding the 3D genome: Emerging impacts on human disease. Seminars in cell \& developmental biology 90, 62-77 (2019).

4. M. J. Fullwood et al., An oestrogen-receptor-alpha-bound human chromatin interactome. Nature 462, 58-64 (2009).

5. E. Lieberman-Aiden et al., Comprehensive mapping of long-range interactions reveals folding principles of the human genome. Science (New York, N.Y.) 326, 289-293 (2009).

6. J. G. Bauman, J. Wiegant, P. Borst, P. van Duijn, A new method for fluorescence microscopical localization of specific DNA sequences by in situ hybridization of fluorochromelabelled RNA. Experimental cell research 128, 485-490 (1980).

7. M. Lakadamyali, M. P. Cosma, Visualizing the genome in high resolution challenges our textbook understanding. Nature methods, 10.1038/s41592-020-0758-3 (2020).

8. C. C. Robinett et al., In vivo localization of DNA sequences and visualization of largescale chromatin organization using lac operator/repressor recognition. The Journal of cell biology 135, 1685-1700 (1996).

9. H. Ma, P. Reyes-Gutierrez, T. Pederson, Visualization of repetitive DNA sequences in human chromosomes with transcription activator-like effectors. Proceedings of the National Academy of Sciences of the United States of America 110, 21048-21053 (2013).

10. R. Ren et al., Visualization of aging-associated chromatin alterations with an engineered

TALE system. Cell research 27, 483-504 (2017).

11. J. D. Sander, J. K. Joung, CRISPR-Cas systems for editing, regulating and targeting genomes. Nature biotechnology 32, 347-355 (2014).

12. B. Chen et al., Dynamic imaging of genomic loci in living human cells by an optimized CRISPR/Cas system. Cell 155, 1479-1491 (2013).

13. M. E. Tanenbaum, L. A. Gilbert, L. S. Qi, J. S. Weissman, R. D. Vale, A protein-tagging system for signal amplification in gene expression and fluorescence imaging. Cell 159, 635-646 (2014).

14. H. Ma et al., Multiplexed labeling of genomic loci with dCas 9 and engineered sgRNAs using CRISPRainbow. Nature biotechnology 34, 528-530 (2016).

15. P. Qin et al., Live cell imaging of low- and non-repetitive chromosome loci using CRISPR-Cas9. Nature communications 8, 14725 (2017).

16. H. Ma et al., CRISPR-Sirius: RNA scaffolds for signal amplification in genome imaging. Nature methods 15, 928-931 (2018).

17. P. G. Maass et al., Spatiotemporal allele organization by allele-specific CRISPR live-cell imaging (SNP-CLING). Nature structural \& molecular biology 25, 176-184 (2018).

18. A. W. Cheng et al., Casilio: a versatile CRISPR-Cas9-Pumilio hybrid for gene regulation and genomic labeling. Cell research 26, 254-257 (2016).

19. Y. Chen, G. Varani, Engineering RNA-binding proteins for biology. The FEBS journal 280, 3734-3754 (2013).

45 20. Y. Wang, C. G. Cheong, T. M. Hall, Z. Wang, Engineering splicing factors with designed specificities. Nature methods 6, 825-830 (2009). 
21. A. Taghbalout et al., Enhanced CRISPR-based DNA demethylation by Casilio-MEmediated RNA-guided coupling of methylcytosine oxidation and DNA repair pathways. Nature communications 10, 4296 (2019).

22. G. Li, Y. Chen, M. P. Snyder, M. Q. Zhang, ChIA-PET2: a versatile and flexible pipeline for ChIA-PET data analysis. Nucleic acids research 45, e4 (2017).

23. J. J. Zhu, A. W. Cheng, JACKIE: Fast enumeration of single- and multi-copy CRISPR binding sites. bioRxiv, 2020.2002.2027.968933 (2020).

24. S. Bae, J. Park, J. S. Kim, Cas-OFFinder: a fast and versatile algorithm that searches for potential off-target sites of Cas9 RNA-guided endonucleases. Bioinformatics (Oxford, England) 30, 1473-1475 (2014).

25. L. Cong et al., Multiplex genome engineering using CRISPR/Cas systems. Science (New York, N.Y.) 339, 819-823 (2013).

26. J. Y. Jeong et al., One-step sequence- and ligation-independent cloning as a rapid and versatile cloning method for functional genomics studies. Applied and environmental microbiology 78, 5440-5443 (2012). 